\title{
Programa computacional para a estimativa da temperatura do ar para a Região Nordeste do Brasil
}

\author{
Enilson P. Cavalcanti', Vicente de P. R. Silva ${ }^{1}$ \& Francisco de A. S. de Sousa ${ }^{1}$
}

\section{RESUMO}

A meta principal através desta pesquisa foi estabelecer modelo de estimativa de temperatura do ar (Estima_T) em função das coordenadas geográficas e das Anomalias de Temperaturas da Superfície do Mar (ATSM). Neste estudo foram utilizadas as séries temporais das médias mensais de temperatura do ar (média diária, mínima e máxima) de 69 estações meteorológicas do Nordeste do Brasil (NEB) e ATSM do oceano Atlântico Tropical. O modelo Estima_T mostrou-se capaz de reconstruir séries temporais de temperatura do ar com razoável precisão para todo o NEB. Os resultados mostraram correlações estatisticamente significantes ao nível de $1 \%$ de probabilidade entre as temperaturas do ar observada e estimada pelo modelo, em toda a região de estudo.

Palavras-chave: Atlântico tropical, Estima_T, ATSM, El Niño e La Niña

\section{A computer program to estimate air temperature for Northeast Region of Brazil}

\begin{abstract}
The objective of this research was to establish a model to estimate air temperature (Estima_T) as a function of geographical coordinates and Sea Surface Temperature Anomalies (SSTA). The mean monthly time series of air temperatures (daily mean, minimum and maximum) of 69 weather stations and SSTA of the Tropical Atlantic were analyzed. The model Estima_T showed good agreement between real and estimated air temperature data of Northeast Brazil. The results showed statistically significant correlation at $1 \%$ level between observed air temperatures and those estimated by model in the whole area of study.
\end{abstract}

Key words: Tropical Atlantic, software Estima_T, SSTA, El Niño and La Niña

' DCA/UFCG. Av. Aprígio Veloso 882, Bodocongó, CEP 58109-970, Fone: (83) 3310-1202. Campina Grande, PB. E-mail: enilson@dca.ufcg.edu.br; vicente@dca.ufcg.edu.br; fassis@dca.ufcg.edu.br 


\section{INTRODUCÃO}

A transferência de calor e massa entre o oceano e a atmosfera é muito dependente da temperatura da superfície do mar e exerce grande influência nas condições de tempo e clima no continente. A baixa amostragem de observações de temperatura do ar em algumas localidades indica a necessidade de se utilizar informações de áreas oceânicas para a obtenção dessa variável meteorológica (Karl et al., 1994).

As águas do oceano Pacífico oriental têm importância fundamental nas variações globais de temperatura, com os eventos refrigerador do La Niña e aquecedor do El Niño. O clima da região Nordeste do Brasil (NEB) é fortemente influenciado pelas Anomalias de Temperaturas da Superfície do Mar (ATSM), sobretudo com estabelecimento do dipolo do oceano Atlântico (Moura \& Shukla, 1981) e do El Niño (Roucou et al., 1996). Outros estudos têm evidenciado teleconexões com Temperaturas da Superfície do Mar (TSM) em várias partes do mundo. Hong et al. (2001) estabeleceram o relacionamento entre o fenômeno El Niño/Oscilação Sul (ENSO) com TSM do Japão; Lentini et al. (2001) investigaram as variabilidades espacial e temporal da TSM na região do Oceano Atlântico Sul; Konda et al. (2002) associaram a TSM com variações sazonais das circulações de monsões na Índia. O efeito do fenômeno El Niño-Oscilação Sul (ENSO) nas temperaturas do ar, global e hemisférica, também tem sido analisado (Privalsky \& Jensen, 1995).

Devido à sua grande extensão e localização, o NEB sofre a influência de vários sistemas atmosféricos, dentre os quais se destacam a zona de convergência intertropical, ondas de leste, frentes frias, brisas e vórtices ciclônicos de ar superior (Roucou et al., 1996). Essa região se caracteriza pelos baixos níveis pluviais, temperaturas elevadas e altas taxas de evaporação. A alta demanda atmosférica produz taxas de evaporação que podem superar até $10 \mathrm{~mm}$ por dia, enquanto a temperatura média do ar varia entre 16,8 a $33,8{ }^{\circ} \mathrm{C}$. $\mathrm{O}$ NEB ocupa uma área de aproximadamente 1,5 milhão de quilômetros quadrados e fica localizado entre os meridia$\operatorname{nos} 48^{\circ} 05^{\prime} \mathrm{W}-35^{\circ} 02^{\prime} \mathrm{W}$ e paralelos $1^{\circ} \mathrm{S}-18^{\circ} 05^{\prime} \mathrm{S}$. Essa extensa região é banhada, ao Norte e a Leste, pelo oceano Atlântico.

A precipitação pluvial em regiões tropicais é fortemente variável no tempo, espaço, duração e quantidade, enquanto a temperatura do ar apresenta baixa variabilidade sendo, portanto, facilmente modelada em função das coordenadas geográficas. $\mathrm{Na}$ ausência de dados observados, ajustes de equações polinomiais têm sido comumente utilizados para se obter a temperatura do ar, em particular, através do método dos mínimos quadrados; entretanto, essas equações têm a capacidade de prever apenas as médias mensais de temperatura do ar em função da elevação do local e das coordenadas geográficas (Cavalcanti \& Silva, 1994; Bolstad et al., 1998).

Como os dados de temperatura do ar são mais escassos e difíceis de obter-se que os da temperatura da água do mar, os cientistas que monitoram o clima da terra têm utilizado as temperaturas da superfície do mar em substituição à temperatura do ar, assumindo que as duas temperaturas aumentam e diminuem proporcionalmente. O conhecimento da tem- peratura do ar é fundamental em várias áreas de pesquisa, principalmente em meteorologia, oceanografia, climatologia e hidrologia. Entretanto, em sua maioria os modelos de estimativa de temperatura do ar dão apenas a média climatológica mensal, os quais não expressam a variação desse elemento climático ao longo do tempo. Objetivou-se, com este trabalho, investigar a ligação entre a TSM e a temperatura do ar no NEB e estabelecer modelo de estimativa da temperatura do ar em função das ATSM e das coordenadas geográficas, para essa região.

\section{MATERIAL E MÉTODOS}

Nesse estudo foram utilizadas as médias mensais de 69 séries temporais de temperatura do ar (média diária, máxima e mínima) do Nordeste do Brasil (Tabela 1) e, também, as séries temporais mensais de Anomalias de Temperaturas da Superfície do Mar (ATSM).

Os dados de ATSM do oceano Atlântico Tropical, obtidos junto ao NOAA (2003), e de temperatura do ar referem-se ao período de 1961 a 1990. As séries temporais das médias mensais de temperatura do ar (média diária, mínima e máxima) das 69 estações meteorológicas da região de estudo, foram reconstruídas em função das coordenadas geográficas (i.e., latitude, longitude e elevação) e das ATSM. O modelo empírico de estimativa da temperatura do ar é uma superfície quadrática, dada por:

$$
\begin{gathered}
\mathrm{T}_{\mathrm{ij}}=\mathrm{a}_{0}+\mathrm{a}_{1} \lambda+\mathrm{a}_{2} \varphi+\mathrm{a}_{3} \mathrm{~h}+\mathrm{a}_{4} \lambda^{2}+\mathrm{a}_{5} \varphi^{2}+\mathrm{a}_{6} \mathrm{~h}^{2}+ \\
\mathrm{a}_{7} \lambda \varphi+\mathrm{a}_{8} \lambda \mathrm{h}+\mathrm{a}_{9} \varphi \mathrm{h}+\operatorname{ATSM}_{\mathrm{ij}}
\end{gathered}
$$

em que $\lambda$ é longitude, $\varphi$ é a latitude, em graus, $\mathrm{h}$ é a elevação de cada estação meteorológica analisada, em metros, e $\mathrm{a}_{0}, \mathrm{a}_{1}, \ldots, \mathrm{a}_{9}$ são os coeficientes de regressão. Os índices i e j indicam, respectivamente, o mês e o ano para o qual se está calculando a temperatura do ar $\left(\mathrm{T}_{\mathrm{ij}}\right)$. Assim, o sinal da ATS$\mathrm{M}_{\mathrm{ij}}$ assume valores positivos e negativos de acordo com o padrão de comportamento de TSM do oceano. Cavalcanti \& Silva (1994) também utilizaram uma superfície quadrática para determinar as temperaturas médias e extremas no Nordeste do Brasil expressa, porém, apenas em função das coordenadas geográficas.

O modelo Estima_T foi desenvolvido em linguagem computacional Visual Basic, para ambiente Windows, podendo ser operado em Pentium PC, com $150 \mathrm{MHz}$ ou superior e $32 \mathrm{Mb}$ de memória RAM. A estimativa da temperatura do ar para a Região Nordeste do Brasil foi feita subdividindo-se a região em três áreas: 1: Maranhão e Piauí; 2: Ceará, Rio Grande do Norte, Paraíba e Pernambuco, e 3: Alagoas; Sergipe e Bahia. Para cada uma dessas áreas determinaram-se os coeficientes da função quadrática para as temperaturas média, máxima e mínima mensal, em função das coordenadas locais (longitude, latitude e altitude), conforme proposto por Cavalcanti \& Silva (1994). A estimativa da série temporal de temperatura do ar (média, máxima e mínima) é obtida adicionando-se, ao valor médio 
Tabela 1. Coordenadas geográficas das estações meteorológicas do Nordeste do Brasil utilizadas no estudo. Todas as estações são localizadas nas latitudes Sul e as longitudes são Oeste, expressas em graus e minutos e as elevações em metro

\begin{tabular}{|c|c|c|c|c|c|c|c|c|c|}
\hline $\mathrm{N}^{0}$ & Estação & Lat. & Long. & Elev. & $\mathrm{N}^{0}$ & Estação & Lat. & Long. & Elev. \\
\hline 1 & Turiacu & $01^{\circ} 43^{\prime}$ & $45^{\circ} 24^{\prime}$ & 44 & 36 & Recife & $08^{\circ} 02^{\prime}$ & $34^{\circ} 53^{\prime}$ & 4 \\
\hline 2 & São Luiz & $02^{\circ} 32^{\prime}$ & $44^{\circ} 18^{\prime}$ & 50 & 37 & Paulistana & $08^{\circ} 20^{\prime}$ & $41^{\circ} 09^{\prime}$ & 350 \\
\hline 3 & Parnaíba & $02^{\circ} 55^{\prime}$ & $41^{\circ} 47^{\prime}$ & 15 & 38 & Arcoverde & $08^{\circ} 26^{\prime}$ & $37^{\circ} 04^{\prime}$ & 663 \\
\hline 4 & Sobral & $03^{\circ} 42^{\prime}$ & $40^{\circ} 21^{\prime}$ & 75 & 39 & Pesqueira & $08^{\circ} 22^{\prime}$ & $36^{\circ} 42^{\prime}$ & 650 \\
\hline 5 & Zé Doca & $03^{\circ} 43^{\prime}$ & $45^{\circ} 32^{\prime}$ & 528 & 40 & Cabrobó & $08^{\circ} 30^{\prime}$ & $39^{\circ} 19^{\prime}$ & 350 \\
\hline 6 & Fortaleza & $03^{\circ} 44^{\prime}$ & $38^{\circ} 32^{\prime}$ & 26 & 41 & Floresta & $08^{\circ} 36^{\prime}$ & $38^{\circ} 35^{\prime}$ & 317 \\
\hline 7 & Carolina & $07^{\circ} 20^{\prime}$ & $47^{\circ} 28^{\prime}$ & 192 & 42 & Garanhuns & $08^{\circ} 52^{\prime}$ & $36^{\circ} 29^{\prime}$ & 866 \\
\hline 8 & Caxias & $04^{\circ} 52^{\prime}$ & $43^{\circ} 21^{\prime}$ & 103 & 43 & Alto Parnaíba & $09^{\circ} 07^{\prime}$ & $45^{\circ} 57^{\prime}$ & 285 \\
\hline 9 & Teresina & $05^{\circ} 04^{\prime}$ & $42^{\circ} 49^{\prime}$ & 72 & 44 & P. de Pedras & $09^{\circ} 10^{\prime}$ & $35^{\circ} 18^{\prime}$ & 22 \\
\hline 10 & Morada Nova & $05^{\circ} 06^{\prime}$ & $38^{\circ} 23^{\prime}$ & 50 & 45 & Caracol & $09^{\circ} 17^{\prime}$ & $43^{\circ} 19^{\prime}$ & 556 \\
\hline 11 & Macau & $05^{\circ} 07^{\prime}$ & $36^{\circ} 38^{\prime}$ & 2 & 46 & Água Branca & $09^{\circ} 17^{\prime}$ & $37^{\circ} 56^{\prime}$ & 510 \\
\hline 12 & Crateús & $05^{\circ} 11^{\prime}$ & $40^{\circ} 40^{\prime}$ & 275 & 47 & Paulo Afonso & $09^{\circ} 21^{\prime}$ & $38^{\circ} 15^{\prime}$ & 250 \\
\hline 13 & Quixeramobim & $05^{\circ} 12^{\prime}$ & $39^{\circ} 18^{\prime}$ & 187 & 48 & Petrolina & $09^{\circ} 23^{\prime}$ & $40^{\circ} 30^{\prime}$ & 376 \\
\hline 14 & Mossoró & $05^{\circ} 12^{\prime}$ & $37^{\circ} 21^{\prime}$ & 15 & 49 & P. dos Índios & $09^{\circ} 24^{\prime}$ & $36^{\circ} 39^{\prime}$ & 342 \\
\hline 15 & Barra do Corda & $05^{\circ} 30^{\prime}$ & $45^{\circ} 16^{\prime}$ & 153 & 50 & Remanso & $09^{\circ} 41^{\prime}$ & $42^{\circ} 04^{\prime}$ & 378 \\
\hline 16 & Imperatriz & $05^{\circ} 32^{\prime}$ & $47^{\circ} 30^{\prime}$ & 123 & 51 & Coruripe & $10^{\circ} 07^{\prime}$ & $36^{\circ} 10^{\prime}$ & 10 \\
\hline 17 & Apodi & $05^{\circ} 40^{\prime}$ & $37^{\circ} 48^{\prime}$ & 305 & 52 & Propriá & $10^{\circ} 13^{\prime}$ & $36^{\circ} 50^{\prime}$ & 17 \\
\hline 18 & Natal & $05^{\circ} 48^{\prime}$ & $35^{\circ} 13^{\prime}$ & 8 & 53 & S. do Bonfim & $10^{\circ} 27^{\prime}$ & $40^{\circ} 11^{\prime}$ & 544 \\
\hline 19 & Tauá & $06^{\circ} 01^{\prime}$ & $40^{\circ} 26^{\prime}$ & 356 & 54 & Aracaju & $10^{\circ} 54^{\prime}$ & $37^{\circ} 03^{\prime}$ & 3 \\
\hline 20 & Colinas & $06^{\circ} 03^{\prime}$ & $44^{\circ} 15^{\prime}$ & 179 & 55 & Barra & $11^{\circ} 50^{\prime}$ & $43^{\circ} 09^{\prime}$ & 410 \\
\hline 21 & Florânia & $06^{\circ} 07^{\prime}$ & $36^{\circ} 49^{\prime}$ & 210 & 56 & Jacobina & $11^{\circ} 10$ & $40^{\circ} 31^{\prime}$ & 460 \\
\hline 22 & Iguatu & $06^{\circ} 22^{\prime}$ & $39^{\circ} 18^{\prime}$ & 213 & 57 & Itabaianinha & $11^{\circ} 16^{\prime}$ & $37^{\circ} 47^{\prime}$ & 225 \\
\hline 23 & Cruzeta & $06^{\circ} 25^{\prime}$ & $36^{\circ} 47^{\prime}$ & 140 & 58 & Irecê & $11^{\circ} 18^{\prime}$ & $41^{\circ} 52^{\prime}$ & 722 \\
\hline 24 & Floriano & $06^{\circ} 46^{\prime}$ & $43^{\circ} 01^{\prime}$ & 85 & 59 & M. do Chapéu & $11^{\circ} 32^{\prime}$ & $41^{\circ} 08^{\prime}$ & 1012 \\
\hline 25 & São Gonçalo & $05^{\circ} 20^{\prime}$ & $38^{\circ} 44^{\prime}$ & 120 & 60 & Alagoinhas & $12^{\circ} 08^{\prime}$ & $38^{\circ} 25^{\prime}$ & 140 \\
\hline 26 & Patos & $07^{\circ} 01^{\prime}$ & $37^{\circ} 17^{\prime}$ & 250 & 61 & Itaberaba & $12^{\circ} 32^{\prime}$ & $40^{\circ} 18^{\prime}$ & 270 \\
\hline 27 & Campos Sales & $07^{\circ} 04^{\prime}$ & $40^{\circ} 23^{\prime}$ & 551 & 62 & Lençóis & $12^{\circ} 34^{\prime}$ & $41^{\circ} 23^{\prime}$ & 394 \\
\hline 28 & Picos & $07^{\circ} 04^{\prime}$ & $41^{\circ} 28^{\prime}$ & 195 & 63 & Salvador & $13^{\circ} 01^{\prime}$ & $38^{\circ} 31^{\prime}$ & 10 \\
\hline 29 & João Pessoa & $07^{\circ} 07^{\prime}$ & $34^{\circ} 53^{\prime}$ & 5 & 64 & B. J. da Lapa & $13^{\circ} 16^{\prime}$ & $43^{\circ} 25^{\prime}$ & 435 \\
\hline 30 & C. Grande & $07^{\circ} 13^{\prime}$ & $35^{\circ} 52^{\prime}$ & 508 & 65 & Itirucu & $13^{\circ} 32^{\prime}$ & $40^{\circ} 08^{\prime}$ & 820 \\
\hline 31 & Barbalha & $07^{\circ} 19^{\prime}$ & $39^{\circ} 19^{\prime}$ & 405 & 66 & Caetite & $14^{\circ} 04^{\prime}$ & $42^{\circ} 29^{\prime}$ & 826 \\
\hline 32 & Balsas & $07^{\circ} 53^{\prime}$ & $46^{\circ} 03^{\prime}$ & 259 & 67 & Carinhanha & $14^{\circ} 18^{\prime}$ & $43^{\circ} 46^{\prime}$ & 452 \\
\hline 33 & Triunfo & $07^{\circ} 50^{\prime}$ & $38^{\circ} 07^{\prime}$ & 1010 & 68 & Ilheus & $14^{\circ} 48^{\prime}$ & $39^{\circ} 18^{\prime}$ & 45 \\
\hline 34 & Surubim & $07^{\circ} 50^{\prime}$ & $35^{\circ} 45^{\prime}$ & 380 & 69 & Canavieiras & $15^{\circ} 40^{\prime}$ & $38^{\circ} 57^{\prime}$ & 4 \\
\hline 35 & Monteiro & $07^{\circ} 53^{\prime}$ & $37^{\circ} 07^{\prime}$ & 590 & & & & & \\
\hline
\end{tabular}

estimado, a anomalia de temperatura do oceano Atlântico Tropical do mês e ano considerado. O programa computacional Estima_T encontra-se disponibilizado gratuitamente na website do Departamento de Ciências Atmosféricas (DCA), da Universidade Federal de Campina Grande, no endereço: http://www.dca.ufcg.edu.br/estima_t/.

Determinou-se o coeficiente de correlação de Pearson, coeficiente de determinação, coeficiente de eficiência, erro médio quadrático e a raiz do erro médio quadrático para avaliar o ajuste entre as séries temporais de temperatura do ar, observadas e obtidas pelo modelo de estimativa da temperatura do ar (Estima_T). Por outro lado, foi empregado o t-test para avaliar a significância estatística dos coeficientes de correlações aos níveis de probabilidade de 1 e 5\%.

\section{RESULTADOS E DISCUSSÃO}

A distribuição espacial dos coeficientes de correlação entre as séries temporais das médias mensais de temperatura do ar (média diária, máxima e mínima) simuladas pelo modelo Estima_T e observadas no Nordeste do Brasil é apresentada na Figura 1. As áreas sombreadas representam os coeficientes de correlação superior a 0,8 , que são estatisticamente significantes ao nível de $1 \%$ de probabilidade, de acordo com o t-test. Privalsky \& Jensen (1995) mostraram relacionamento linear entre índice de oscilação sul e temperatura global do ar, estatisticamente significante ao nível de 10\%. Cavalcanti \& Silva (1994), quando ajustaram uma superfície quadrática em função das coordenadas geográficas, com o objetivo de estimarem a temperatura média do ar no NEB, obtiveram coeficiente de correlação que variaram de 0,76 a 0,99 . De acordo com a figura mencionada, a correlação é maior na costa Leste no Nordeste do Brasil (i.e., entre os meridianos $40-35^{\circ} \mathrm{W}$ ) e menor na costa Norte, exceto a temperatura máxima (Figura 1c), que apresenta coeficiente de correlação superior a 0,8 nos litorais Norte e Leste e núcleos de 0,7 no sudoeste do Estado da Bahia. As temperaturas média diárias, máxima e mínima nesse Estado, foram as que apresentaram menores correlações com os dados do modelo, cujo resultado sugere que a advecção de calor sensível no sistema oceano-terra (isto é, o aquecimento ou esfriamento no continente) é afetado pela distância entre as áreas inter-relacionadas e pela circulação local de ventos.

A faixa que compreende o litoral Norte da região de estudo 
A.

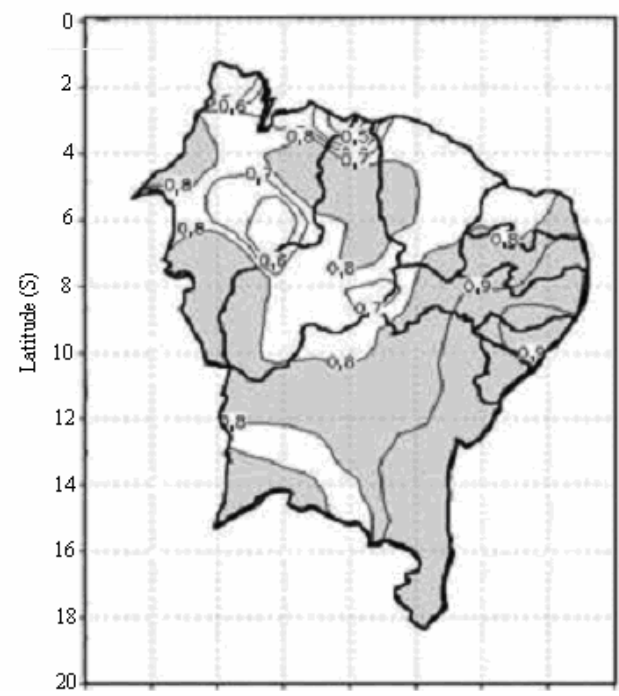

B.

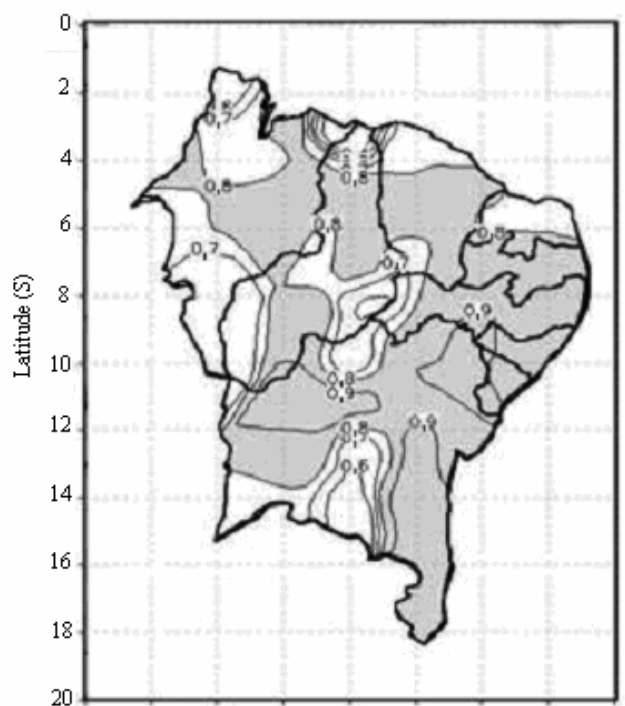

C.

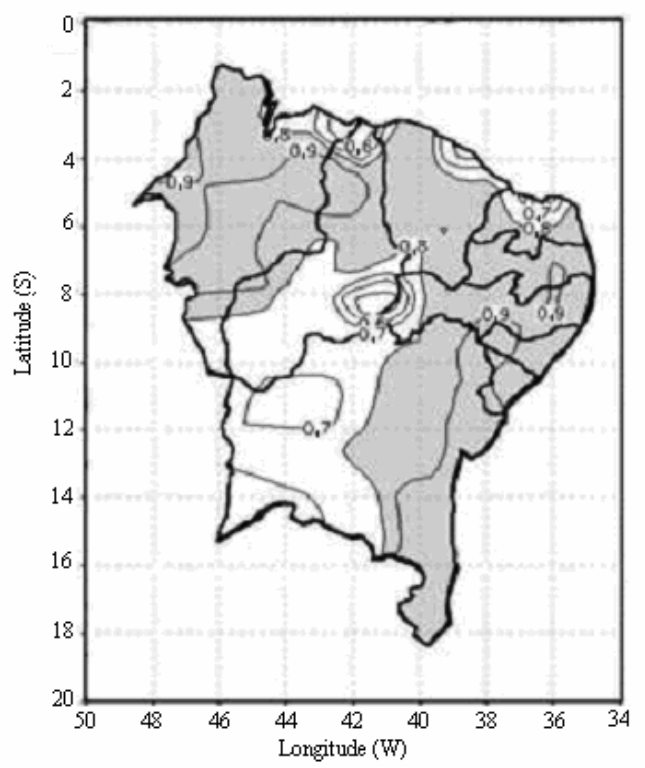

Figura 1. Distribuição espacial dos coeficientes de correlação entre as séries temporais de temperatura do ar (1961-1990) observada e simulada pelo modelo Estima_T: A) temperatura média, B) temperatura mínima e C) temperatura máxima exibe alguns núcleos de correlações inferiores a 0,8 entre temperatura do ar média diária com a temperatura estimada com base no modelo (Figura 1A), e com a temperatura mínima do ar (Figura 1B). Essa mesma faixa do NEB mostra correlações superiores a 0,8 quando a associação é feita entre temperatura máxima do ar observada e estimada com base no modelo Estima-T (Figura 1A). Muitos pesquisadores têm avaliado a influência de fenômenos físicos de diversas naturezas sobre a temperatura do ar. Por exemplo, Butler \& Johnston (1996) confirmaram a ligação entre a atividade solar e temperatura média do ar; Privalsky \& Jensen (1995) avaliaram a influência do fenômeno ENSO sobre a temperatura global do ar; Vadász (1994) estudou o relacionamento da temperatura do ar e do índice de vegetação e Wilson (1999) analisou a correlação entre a temperatura do ar e El Niño e erupções vulcânicas.

A Tabela 2 exibe os valores de algumas estatísticas que indicam o grau de adequabilidade entre valores observados e estimados pelo modelo. Estas estatísticas revelam ótimo ajuste entre os dados, exceto em algumas estações localizadas ao Norte da região de estudo, tais como Floriano (PI) e São Luiz (MA). Observa-se que o coeficiente de determinação $\left(\mathrm{r}^{2}\right)$ foi superior a $70 \%$ na grande maioria das estações analisadas.

A Figura 2 descreve a variação temporal das temperaturas do ar (média, mínima e máxima) observada na localidade de Campina Grande (latitude: $07^{\circ} 13^{\prime} \mathrm{S}$, longitude: $35^{\circ} 52^{\prime} \mathrm{W}$ ) e estimada pelo modelo Estima-T, durante o período 1961-1990. Os dados observados da temperatura do ar se ajustaram muito bem aos dados obtidos com o modelo. O ciclo anual e a tendência crescente da temperatura do ar, constatada nessa localidade, são perfeitamente reproduzidos com os dados do modelo, porém eles apresentam tendência para superestimar os dados observados de temperatura do ar,

Tabela 2. Coeficiente de correlação de Pearson (r), coeficiente de determinação $\left(r^{2}\right)$, coeficiente de eficiência (CE), erro médio quadrático e raiz do erro médio quadrático (REMQ) entre valores observados e estimados pelo modelo

\begin{tabular}{lccccc}
\hline & $\mathbf{r}$ & $\mathbf{r}^{2}$ & CE & EMQ & REMQ \\
Monteiro & 0,8926 & 0,8000 & 0,4752 & 0,7406 & 0,8636 \\
Campina Grande & 0,9158 & 0,8386 & 0,4704 & 0,6804 & 0,8273 \\
Imperatriz & 0,7623 & 0,5811 & 0,2860 & 0,7479 & 0,8834 \\
\hline Apodi & 0,7502 & 0,5628 & 0,1912 & 2,6130 & 1,6216 \\
Arco verde & 0,9190 & 0,8445 & 0,5485 & 0,6143 & 0,7874 \\
Petrolina & 0,8155 & 0,8155 & 0,3710 & 1,4139 & 1,1940 \\
\hline Picos & 0,8451 & 0,7142 & 0,4279 & 0,9066 & 0,9554 \\
Itabaianinha & 0,9351 & 0,8744 & 0,4786 & 0,7351 & 0,8639 \\
Ilhéus & 0,9421 & 0,8876 & 0,5667 & 0,4899 & 0,7053 \\
Sobral & 0,7413 & 0,5495 & 0,2397 & 0,8527 & 0,9262 \\
São Luiz & 0,6898 & 0,4759 & 0,2674 & 0,4114 & 0,6523 \\
Senhor do Bonfim & 0,9038 & 0,8169 & 0,5114 & 0,8014 & 0,8952 \\
Palmeiras dos Índios & 0,8135 & 0,6618 & 0,5312 & 1,2580 & 1,1289 \\
Própria & 0,9339 & 0,8722 & 0,5160 & 0,5875 & 0,0430 \\
\hline Floriano & 0,6795 & 0,4617 & 0,4027 & 3,0489 & 1,7571 \\
\hline Mossoró & 0,7198 & 0,5181 & 0,2861 & 0,5443 & 0,7407 \\
\hline Quixeramobim & 0,8152 & 0,6645 & 0,4246 & 0,5544 & 0,7468 \\
\hline Aracaju & 0,8875 & 0,7877 & 0,5321 & 0,9998 & 1,0076 \\
\hline
\end{tabular}


A.

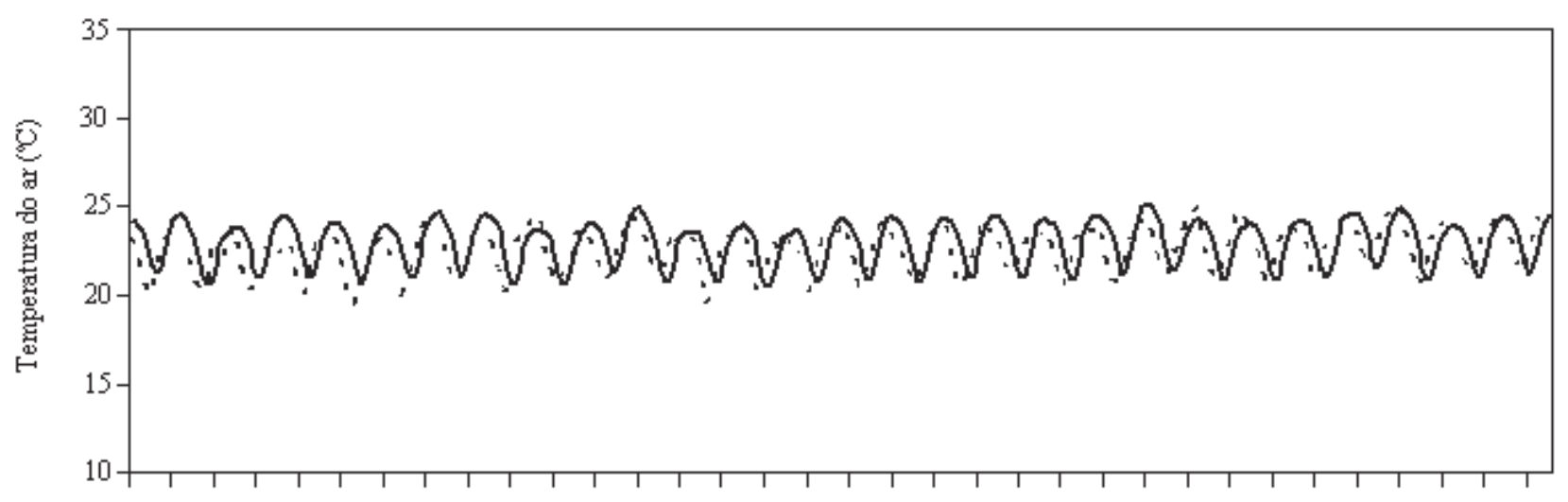

B.

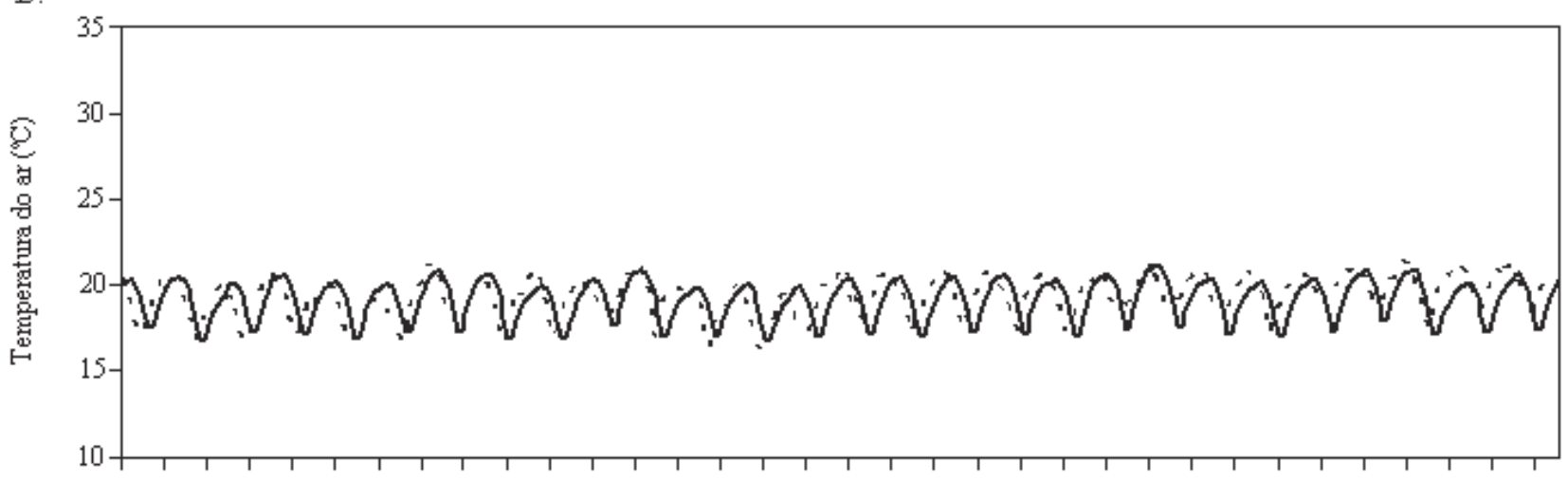

C.

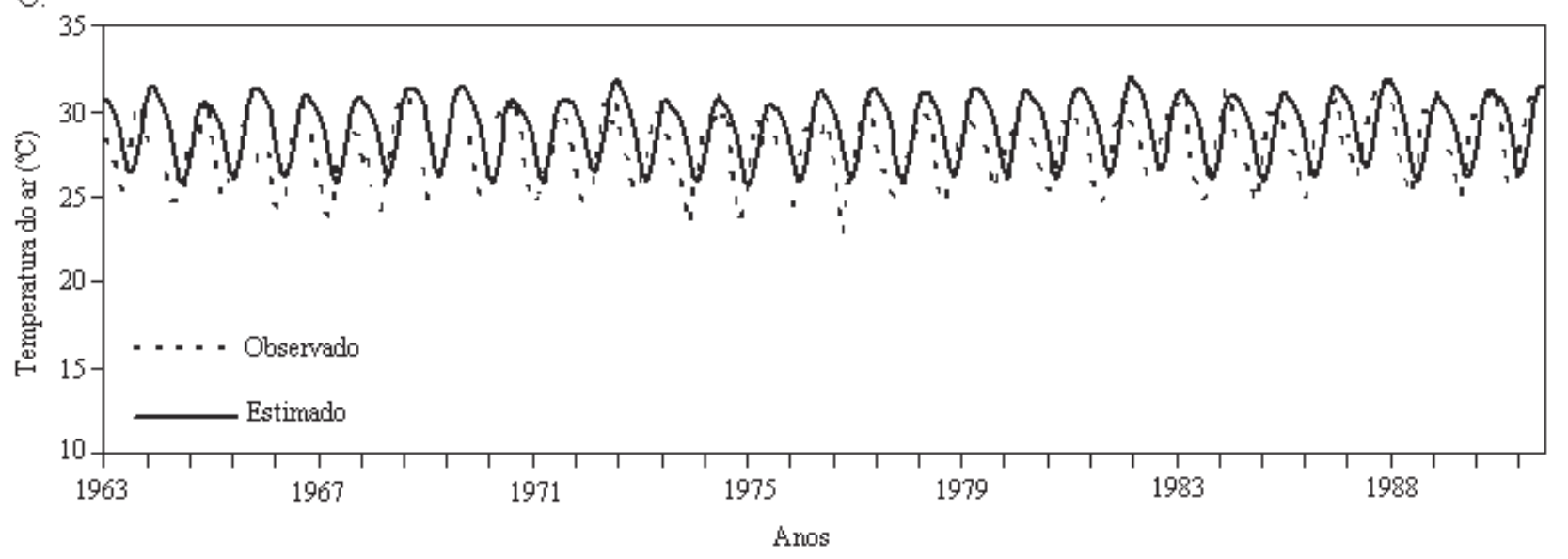

Figura 2. Comparação entre a temperatura média do ar observada (linhas cheias) em Campina Grande $\left(07^{\circ} 13^{\prime} \mathrm{S}-35^{\circ} 52^{\prime} \mathrm{W}\right)$ e simulada pelo modelo Estima-T (linhas pontilhadas): A) temperatura média, B) temperatura mínima e C) temperatura máxima

durante o período analisado.

A relação entre as temperaturas do ar (média diária, mínima e máxima) observadas e estimadas pelo modelo para Campina Grande, PB, é apresentado na Figura 3. As três séries temporais de temperatura do ar exibem coeficientes de determinação relativamente altos, sendo a maior precisão apresentada para a temperatura média do ar (Figura 3A), com $\mathrm{r}^{2}=0,834$, enquanto a menor precisão ocorreu para a temperatura mínima (Figura $3 \mathrm{~B}$ ), com $\mathrm{r}^{2}=0,78$. Esses resultados sugerem que a temperatura do ar (média diária, máxima e mínima) no NEB pode ser prevista com razoável nível de precisão, em função das coordenadas geográficas e das anomali- as de TSM. Sun \& Furbish (1997) também observaram que a precipitação anual e a vazão de rios na Península da Flórida, EUA, podem ser previstas a partir de anomalias de TSM do Pacífico Tropical Leste. Roy \& Reason (2001) utilizando o modelo de correlação linear, encontraram coeficientes de correlação de 0,73 entre ENSO e anomalias de TSM.

\section{Operacionalização do programa computacional}

$\mathrm{Na}$ janela principal do programa computacional Estima_T (Figura 4) define-se o mês e a área desejada para cálculo. As opções de estimativa são: "Postos convencionais": permite que se estime a temperatura do ar média máxima e mínima 

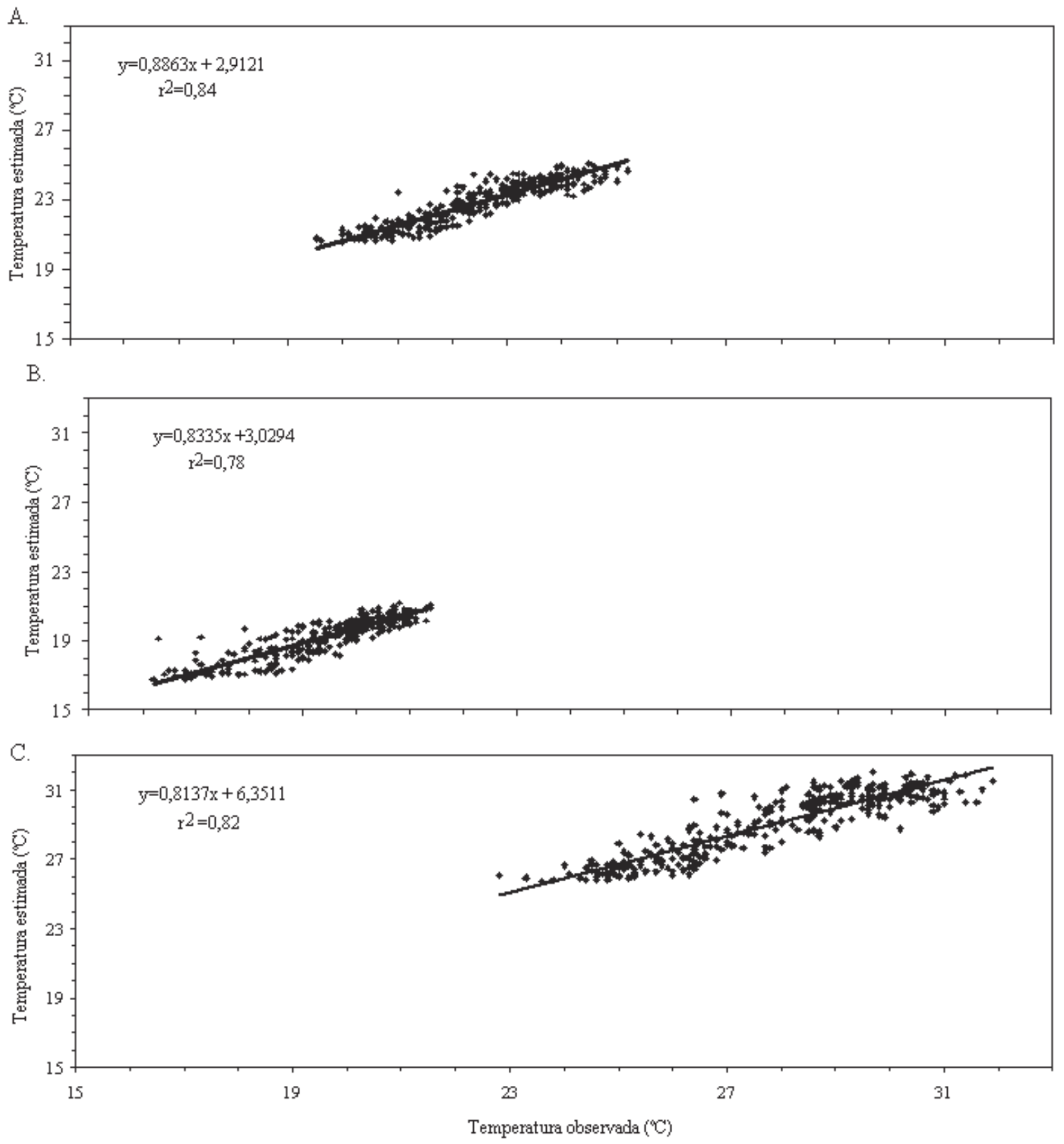

Figura 3. Relacionamento entre as temperaturas do ar $\left({ }^{\circ} \mathrm{C}\right)$ observada e estimada pelo modelo Estima T para a localidade de Campina Grande, $\mathrm{PB}$ : $\left.\mathrm{A}\right)$ temperatura média, B) temperatura mínima e C) temperatura máxima ( $\mathrm{n}=336)$

mensal, para postos pluviométricos já cadastrados no sistema; "Pontos específicos": permite estimar-se a temperatura do ar média máxima e mínima mensal, para pontos estabelecidos pelo usuário e, finalmente, "Estima série": permite se estimar a temperatura do ar média máxima e mínima mensal para determinada localidade, desde 1950, até a presente data. A opção "Postos convencionais" fornece relatório para a área especificada, referente aos postos que formam uma base de dados do modelo Estima_T (Figura 5).

Na opção "Postos específicos", abre-se uma janela que permite direcionar o arquivo de leitura referente ao cadastro preparado pelo usuário (*.dat). Esse arquivo cadastro é cons- truído colocando-se, em cada linha, a seqüência: longitude, latitude, altitude e nome do posto. Na primeira linha do arquivo deve-se definir o número total de posto cadastrado. A leitura de arquivo específico preparado pelo usuário pode ser localizada conforme exibe a Figura 6. Na opção "Estima série" é possível abrir uma janela (Figura 7) para se escolher as coordenadas geográficas de uma localidade qualquer da área especificada. Os limites estabelecidos devem ser obedecidos em qualquer estimativa. Ao serem efetuados os cálculos, será emitido relatório (Figura 8) com as temperaturas média, máxima e mínima, mês a mês, para a localidade escolhida de janeiro de 1950 até a data mais recente da 


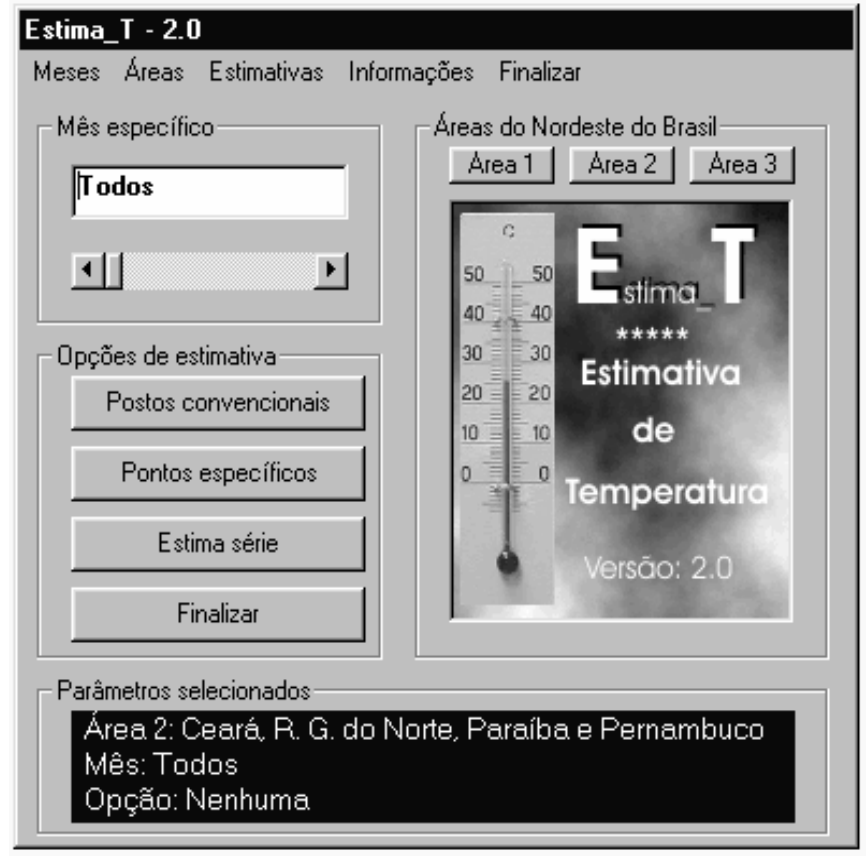

Figura 4. Tela principal do programa computacional Estima_T com as opções de cálculo: Pontos convencionais, Pontos específicos e Esstima série

\begin{tabular}{|c|c|c|c|c|c|}
\hline \multirow{2}{*}{\multicolumn{6}{|c|}{ Wf Estima_T - 2.0 - Relatório: dados.txt }} \\
\hline & & & & & \\
\hline \multicolumn{6}{|c|}{$\begin{array}{l}\text { Área 2: Ceará, R. G. do Norte, Paraíba e Pernambuco } \\
\text { Sequencia: Longitude, Latitude, Altitude e Temperaturas (C) }\end{array}$} \\
\hline \multicolumn{2}{|c|}{ Temperatura Média } & \multicolumn{3}{|c|}{ Mês: janeiro } & \\
\hline$-40,00$ & $-02,00$ & 0007,00 & 25,53 & I & ACARAU - CE \\
\hline$-41,00$ & $-03,00$ & 0685,00 & 21,01 & $\mathrm{i}$ & VICOSA DO CEARA \\
\hline$-40,00$ & $-03,00$ & 0084,00 & 26,33 & j & SOBRAL - CE \\
\hline$-38,00$ & $-03,00$ & 0027,00 & 26,41 & i & FORTALEZA - CE \\
\hline$-39,02$ & $-04,97$ & 0180,00 & 27.56 & j & QUIXADA - CE \\
\hline$-38,95$ & $-04,27$ & 0870,67 & 21,44 & 1 & GUARAMIRANGA - \\
\hline$-37,77$ & $-04,57$ & 0013,00 & 28,06 & $\mathrm{i}$ & ARACATI-FORTIM - \\
\hline$-40,67$ & $-05,18$ & 0276,75 & 26,85 & 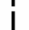 & CRATEUS - CE \\
\hline$-39,30$ & $-05,20$ & 0211,09 & 27.53 & j & QUIXERAMOBIM - C \\
\hline$-38,38$ & $-05,10$ & 0050,00 & 28,49 & i & MORADA NOVA - C \\
\hline$-40,42$ & $-06,02$ & 0399,59 & 26,42 & $\mathrm{i}$ & TAUA - CE \\
\hline$-39,30$ & $-06,37$ & 0211,69 & 28,18 & j & IGUATU - CE \\
\hline
\end{tabular}

Figura 5. Relatório da estimativa da temperatura do ar para postos da área 2: Ceará, Rio Grande do Norte, Paraíba e Pernambuco

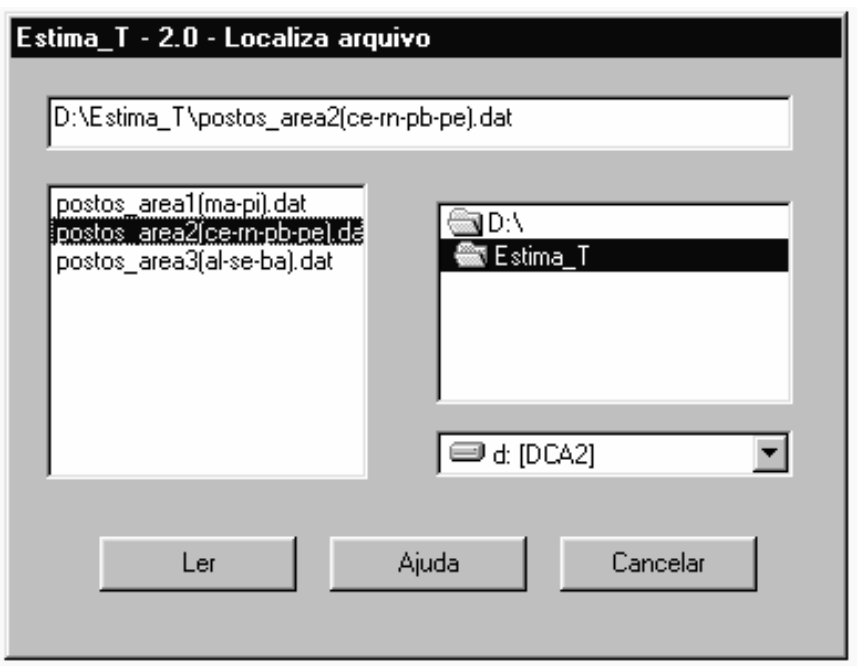

Figura 6. Leitura do arquivo específico preparado pelo usuário do programa computacional Estima_T

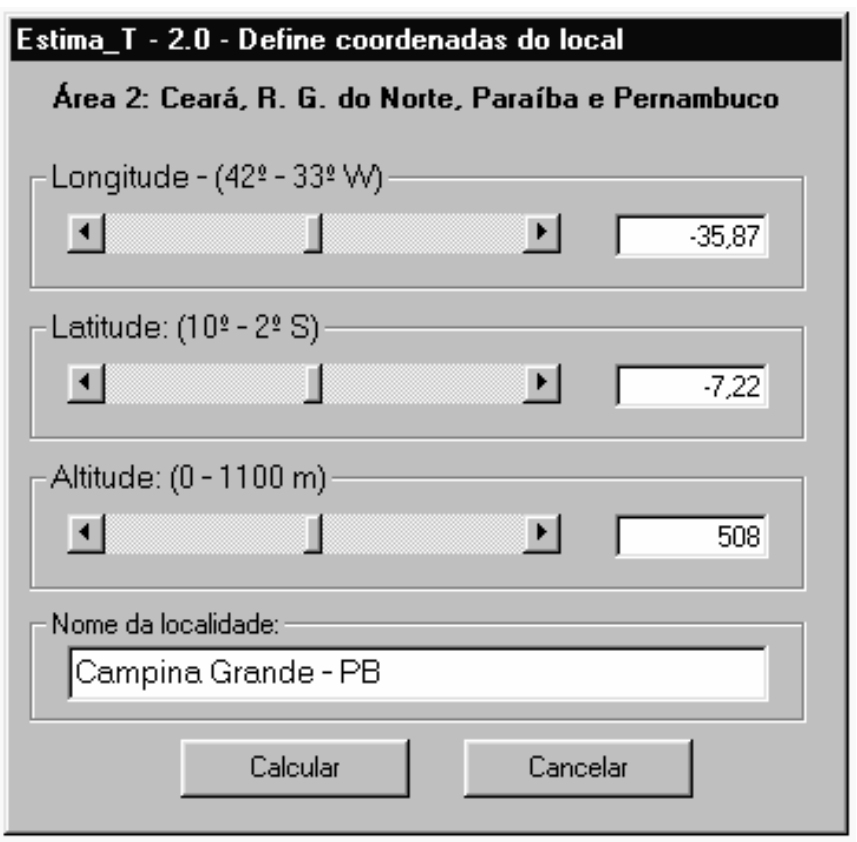

Figura 7. Seleção das coordenadas geográficas da localidade analisada

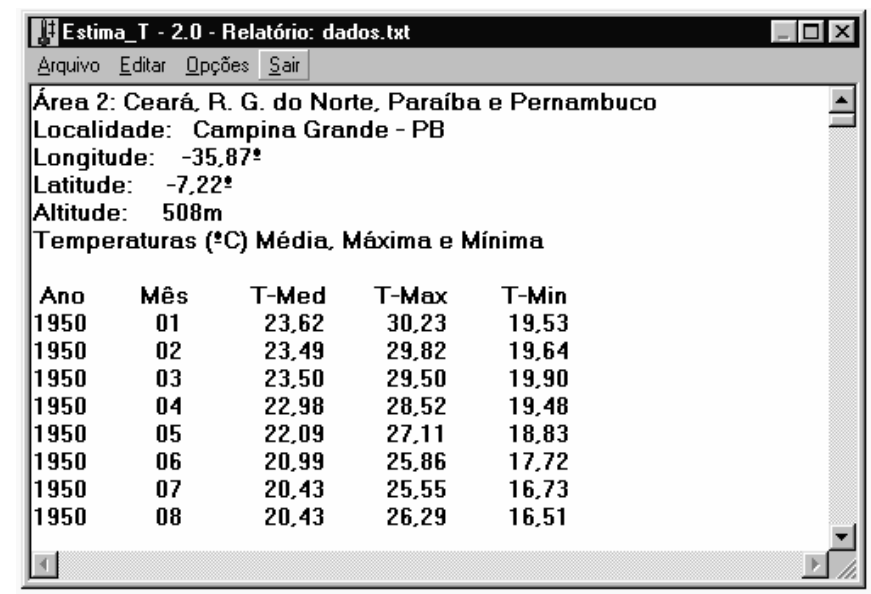

Figura 8. Relatório da série temporal estimada para a localidade de Campina Grande, Paraíba

atualização das Anomalias da Temperatura da Superfície do Mar (ATSM) do Oceano Atlântico Tropical.

\section{CONCLUSÕES}

1. O modelo de previsão apresentado neste estudo pode ser utilizado com razoável nível de precisão para construção de séries temporais de temperatura do ar no NEB.

2. A temperatura do ar (média diária, máxima e mínima) de todo Litoral Leste do NEB, que compreende a extensa área entre os meridianos $40^{\circ}-35^{\circ} \mathrm{W}$, é fortemente influenciada pelas anomalias de TSM do oceano Atlântico Tropical.

3. As séries temporais de temperaturas do ar (média diária) observada e estimada pelo modelo Estima_T na localidade de Campina Grande mostraram comportamento temporal semelhantes, inclusive com suave tendência crescente durante o período analisado. 


\section{LITERATURA CITADA}

Bolstad, P. V.; Swift, L.; Collins, F.; Régnière, J. Measured and predicted air temperature at basin to regional scales in the southem Appalachian mountains. Agricultural and Forest Meteorology, Amsterdam, v.91, n.2-4, p.161-176, 1998.

Butler, C. J.; Johnston, D. J. A provisional long mean air temperature series for Armagh Observatory. Joumal of Atmospheric and Solar-Terrestrial Physics, Oxford, v.58, n.15,p.1657-1672, 1996 .

Cavalcanti, E. P.; Silva, E. D. V. Estimativa da temperatura do ar em função das coordenadas locais. In: Congresso Brasileiro de Meteorologia, 7, Congresso Latino-Americano e Ibérico de Meteorologia, 2, 1994, Belo Horizonte, Anais...SBM, p.154-157. 1994.

Hong, Chul-hoon; Cho, Kya-Dae; Kim, Hee-Joon. The relationship between ENSO events and sea surface temperature in the East (Japan) Sea. Progress in Oceanography, Oxford, v.49, n.1-4, p.21-40, 2001.

Karl, T. R.; Knight, R. W.; Christy, J. R. Global and hemispheric temperature trends: Uncertainties related to inadequate spatial sampling. Journal of Climate, Boston, v.7, n.1, p.1114-1163, 1994.

Konda, M.; Imasato, N.; Shibata, A. Interannual variability of the sea-surface temperature in the Indian Ocean in response to the air-sea turbulent heat exchange. Deep-Sea Research II, Oxford, v.49, n.7-8, p.1527-1548, 2002.

Lentini, C. A. D.; Podestá, G. G.; Campos, E. J. D.; Olson, D. B. Sea surface temperature anomalies on the Western South Atlantic from 1982 to 1994. Continental Shelf Research, Oxford, v.21, n.1, p.89-112, 2001.
Moura, A. D.; Shukla, J. On the dynamics of droughts in North-East Brazil: observations, theory and numerical experiments with a general circulation model. Journal of the Atmospheric Science, Boston, v.38, n.1,p.2653-2675, 1981.

NOAA. Climate Prediction Center/Monthly Atmospheric Indices. http:// www.cpc.noaa.gov/data/indices/index.html., acesso em:10 Jun. 2003.

Privalsky, V.E.; Jensen, D.T. Assessment of the influence of ENSO on annual global temperature. Dynamics of Atmospheres and Oceans, Amsterdam, v.22, n.1, p.161-178, 1995.

Roucou, P.; Aragão, J. O. R.; Harzallah, A.; Fontaine, B.; Janicot, S. Vertical motion, changes to Northeast Brazil rainfall variability: A GCM simulation. International Journal of Climatology, Chichester, v.16, n.1, p.879-891, 1996.

Roy, C.; Reason, C. ENSO related modulation of coastal upwelling in the eastern Atlantic. Progress in Oceanography, Oxford, v.49, n.14,p.245-255, 2001.

Sun, H.; Furbish, D. J. Annual precipitation and river discharges in Florida in response to El Niño-and La Niña-sea surface temperature anomalies. Journal of Hydrology, Amsterdam, v.199, n.1-2,p.74-87, 1997.

Vadász, V. On the relationship between surface temperature, air temperature and vegetation index. Advanced Space Research, Oxford, v.14, n.3, p.41-44, 1994.

Wilson, R. M. Variation of surface air temperature in relation to El Niño and cataclysmic volcanic eruptions, 1796-1882. Journal of Atmospheric and Solar-Terrestrial Physics, Oxford, v.61, v.1, p.1307-1319, 1999. 